\title{
Treatment Effects of Xuebijing Injection in Severe Septic Patients with Disseminated Intravascular Coagulation
}

\author{
Qin Yin and Chunsheng Li \\ Emergency Department, Beijing Chaoyang Hospital, Capital Medical University, No. 8 Workers' Stadium South Road, \\ Beijing 100020, China
}

Correspondence should be addressed to Chunsheng Li; lcscyyy@163.com

Received 15 December 2013; Revised 18 February 2014; Accepted 19 February 2014; Published 23 March 2014

Academic Editor: Yuping Tang

Copyright (C) 2014 Q. Yin and C. Li. This is an open access article distributed under the Creative Commons Attribution License, which permits unrestricted use, distribution, and reproduction in any medium, provided the original work is properly cited.

\begin{abstract}
The treatment effects of Xuebijing (XBJ) injection in severe septic patients with disseminated intravascular coagulation (DIC) were investigated in this study. 171 severe septic patients with DIC were divided into the control group $(n=83)$ or intervention group $(n=88)$. Routine therapies were administered in both groups, and XBJ injection was administered additionally in the intervention group. Incidence of DIC, clinical severity scores, and coagulation parameters at 7 days after administration of XBJ injection were compared between the two groups, and short-term prognosis was evaluated by 28 -day mortality. Compared with the control group, incidence of DIC in the intervention group was significantly lower at 7 days after administration of XBJ injection $(P<0.001)$. In addition, differences of platelet count and prothrombin time were significantly greater in the intervention group than in the control group $(P$ all $<0.05)$, and similar results were also found for differences of the Mortality in Emergency Department Sepsis score and Acute Physiology and Chronic Health Evaluation II score $(P$ all $<0.05)$. Furthermore, 28-day mortality was significantly lower in the intervention group $(P=0.034)$. These results demonstrate that XBJ injection can effectively treat DIC caused by severe sepsis and improve short-term prognosis of severe septic patients with DIC.
\end{abstract}

\section{Introduction}

Despite advancements in modern antibiotics and supportive therapies, the mortality of severe sepsis remains high. In the United States, severe sepsis accounts for 751,000 hospital admissions and 215,000 deaths every year, with an inhospital mortality of $28.6 \%$ [1]. Disseminated intravascular coagulation (DIC), a serious complication of severe sepsis, is characterized by widespread fibrin deposition in microvessels resulting from coagulation activation, inhibition of anticoagulation and fibrinolysis, and subsequent consumption of clotting factors and hyperfibrinolysis [2]. DIC is closely associated with the development of multiple organ failure, and mortality in patients with DIC is much higher than in those without DIC [3].

Current management of DIC relies on treating the underlying disease aggressively, along with supplementary therapy with clotting factors and platelets as required [4]. In addition, anticoagulation is also the main target of treatment. Heparin is the most widely used anticoagulant in clinical practice, but controlled trials did not confirm the survival benefit of heparin $[5,6]$. Another anticoagulant, recombinant human activated protein C (rhAPC), was reported to decrease mortality in the Recombinant Human Activated Protein C Worldwide Evaluation in Severe Sepsis (PROWESS) study [7], and post hoc data analysis demonstrated that patients with DIC may have a survival benefit in particular [8]. However, this drug was found not to offer a survival benefit in the recent PROWESS-SHOCK study [9]. In the latest guidelines of Surviving Sepsis Campaign, rhAPC has been withdrawn [10]. Taken together, there is an urgent need for new effective therapies for DIC.

Xuebijing (XBJ) injection is an intravenous preparation made from five traditional Chinese medicines, namely, Chishao (Radix Paeoniae Rubra), Danggui (Radix Angelica Sinensis), Chuanxiong (Rhizoma Chuanxiong), Honghua (Flos Carthami), and Danshen (Radix Salviae Miltiorrhizae). The bioactive roles of XBJ injection include activating circulation, removing blood stasis, and clearing away toxins [11]. Previous studies have confirmed that XBJ injection is effective 
for treating sepsis [12], and this drug has been formally approved by the State Food and Drug Administration of China for use in clinical practice.

Although the effects of XBJ injection for treating sepsis have been established, there are few studies on XBJ injection in DIC. Some studies have shown that XBJ injection is also effective in treating patients with DIC [13, 14]. However, the dose and time of XBJ injection were not uniform, and the sample sizes were relatively small in these studies. Therefore, the main purpose of this study was to investigate the treatment effects of XBJ injection on DIC caused by severe sepsis.

\section{Patients and Methods}

2.1. Patients and Settings. This study included 171 adult severe septic patients with DIC who were admitted to the emergency intensive care unit (EICU) of Beijing Chaoyang Hospital, which is an urban, university hospital, from January 2011 to December 2011. Exclusion criteria were as follows: age $<18$ years, survival time $<7$ days after EICU admission, previous history of coagulopathy, use of anticoagulants, pregnancy, or breast feeding.

According to the criteria of the 2001 SCCM/ESICM/ ACCP/ATS/SIS International Sepsis Definitions Conference [15], systemic inflammatory response syndrome (SIRS) was defined with two or more of the following criteria: (1) body temperature $>38.3^{\circ} \mathrm{C}$ or $<36^{\circ} \mathrm{C}$; (2) heart rate $>90$ beats per minute; (3) respiratory rate $>20$ breaths per minute or $\mathrm{PaCO}_{2}<32 \mathrm{mmHg}$; (4) white cell count $>12 \times 10^{9} / \mathrm{L}$ or $<4 \times 10^{9} /$ L or the percentage of immature neutrophil $>10 \%$. Sepsis was defined as SIRS caused by infection. Severe sepsis was defined as sepsis-induced acute organ dysfunction. The criteria of acute organ dysfunction were as follows: (1) sepsis-induced hypotension; (2) lactate above normal upper limits; (3) urine output $<0.5 \mathrm{~mL} / \mathrm{kg} / \mathrm{hour}$ for more than 2 hours despite adequate fluid resuscitation or creatinine $>2.0 \mathrm{mg} / \mathrm{dL}(176.8 \mu \mathrm{mol} / \mathrm{L})$; (4) acute lung injury with $\mathrm{PaO}_{2} / \mathrm{FiO}_{2}<250 \mathrm{mmHg}$ in the absence of pneumonia as infection source or acute lung injury with $\mathrm{PaO}_{2}$ / $\mathrm{FiO}_{2}<200 \mathrm{mmHg}$ in the presence of pneumonia as infection source; (5) bilirubin $>2.0 \mathrm{mg} / \mathrm{dL}(34.2 \mu \mathrm{mol} / \mathrm{L})$; and (6) platelet count $<100,000 \mu \mathrm{L}$ or international normalized ratio $($ INR) $>1.5$.

DIC was diagnosed according to the criteria of the International Society on Thrombosis and Haemostasis Subcommittee. Platelet count, prothrombin time (PT), fibrinogen, and D-dimer levels were used to calculate the DIC score, and a score $\geq 5$ was considered compatible with overt DIC (Table 1) [16].

\subsection{Methods}

2.2.1. Study Design. As this was a retrospective study, ethical approval and informed consent were not necessary according to the Institutional Review Board of Beijing Chaoyang Hospital. The available database of laboratory parameters and the clinical database of our EICU were used in this study.
TABLE 1: ISTH scoring system for overt DIC.

\begin{tabular}{lcccc}
\hline & 0 & 1 point & 2 points & 3 points \\
\hline $\begin{array}{l}\text { Platelet } \\
\left(\times 10^{9} / \mathrm{L}\right)\end{array}$ & $\geq 100$ & $<100$ but $\geq 50$ & $<50$ & \\
$\begin{array}{l}\text { Prolongation } \\
\text { of } \mathrm{PT}\end{array}$ & $\leq 3 \mathrm{sec}$ & $>3 \mathrm{sec}$ but $\leq 6 \mathrm{sec}$ & $>6 \mathrm{sec}$ & \\
$\begin{array}{l}\text { Fibrinogen } \\
(\mathrm{g} / \mathrm{L})\end{array}$ & $\geq 1.0$ & $<1.0$ & & \\
$\begin{array}{l}\mathrm{D} \text {-dimer } \\
(\mathrm{mg} / \mathrm{L})\end{array}$ & $\leq 0.4$ & & $>0.4$ but $\leq 4$ & $>4$ \\
\hline
\end{tabular}

ISTH: International Society on Thrombosis and Haemostasis; DIC: disseminated intravascular coagulation; PT: prothrombin time.

2.2.2. Treatment Regiment. The mainstays of routine treatments for severe septic patients with DIC were antibiotics, fluid resuscitation, mechanical ventilation, vasoactive agents, nutritional support, and transfusion of blood products, whereas coagulation inhibitors and anticoagulants were not used in our EICU. Patients were divided into the control group or intervention group. For patients in the control group, only routine treatments were administered. For patients in the intervention group, besides routine treatments, $100 \mathrm{~mL} \mathrm{XBJ}$ injection (Tianjin Chasesun Pharmaceutical Co., Ltd., Tianjin, China) with $100 \mathrm{~mL}$ normal saline was administered via intravenous drip twice daily for 7 consecutive days. XBJ injection was administered separately, and $50 \mathrm{~mL}$ normal saline was used to wash the pipeline before and after administration of XBJ injection. Therefore, XBJ injection did not interact with other routine medicines.

2.2.3. Evaluation of Treatments. The primary efficacy endpoint was incidence of DIC as evaluated at 7 days after administration of XBJ injection. Other evaluation parameters were as follows: (1) clinical severity scores, including the Mortality in Emergency Department Sepsis (MEDS) score and Acute Physiology and Chronic Health Evaluation (APACHE) II score; (2) coagulation parameters, including platelet, PT, fibrinogen, D-dimer, and INR; and (3) differences of clinical severity scores and coagulation parameters (values at baseline minus values at 7 days after administration of XBJ injection).

Nine variables of the MEDS score were as follows: terminal illness, tachypnea or hypoxia, septic shock, low platelet count, bandemia, age $>65$ years, lower respiratory tract infection, nursing home resident, and altered mental status. The MEDS score was calculated by summing the points of nine variables, giving a possible score of 0 to 27 [17].

The APACHE II score comprised weightings for temperature, mean arterial pressure, heart rate, respiratory rate, oxygenation, arterial $\mathrm{pH}$, sodium, potassium, creatinine, hematocrit, white blood cell count, Glasgow coma score, age, chronic diseases, and surgical status, giving a possible score of 0 to $71[18]$.

PT, INR, fibrinogen, and D-dimer levels were measured on sodium citrate anticoagulated samples using Sysmex CA7000 blood coagulation analyzer (Sysmex Corporation, Kobe, Japan). PT, INR, and plasma fibrinogen levels were 
TABLE 2: Baseline characteristics of patients.

\begin{tabular}{lccc}
\hline & Control group $(n=83)$ & Intervention group $(n=88)$ & $P$ \\
\hline Age $($ yrs $)$ & $56(49-73)$ & $59(53-74)$ & 0.14 \\
Male $(n, \%)$ & $51(61.45)$ & $57(64.77)$ & 0.652 \\
BMI & $23.67 \pm 2.93$ & $22.97 \pm 3.40$ & 0.158 \\
28 -day mortality $(n, \%)$ & $29(34.94)$ & $78(20.45)$ & 0.034 \\
Platelet $\left(\times 10^{9} / \mathrm{L}\right)$ & $79.61 \pm 49.69$ & $22.66 \pm 8.46$ & 0.326 \\
PT $(\mathrm{s})$ & $25.72 \pm 7.34$ & $2.57 \pm 1.93$ & 0.263 \\
Fibrinogen $(\mathrm{g} / \mathrm{L})$ & $2.64 \pm 1.47$ & $5.2(2.12-9.53)$ & 0.17 \\
D-dimer $(\mathrm{mg} / \mathrm{L})$ & $4.7(1.85-8.62)$ & $1.71 \pm 0.88$ & 0.192 \\
INR & $1.79 \pm 0.91$ & $12(9-21)$ & 0.607 \\
MEDS score & $11(8-19)$ & $26(16-35)$ & \\
APACHE II score & $24(15-32)$ & & 0.794 \\
Type of infection $(n, \%)$ & & $49(55.68)$ & 0.334 \\
Pneumonia & $39(46.88)$ & $33(37.5)$ & $6(6.82)$ \\
IAI & $37(44.58)$ & $7(8.43)$ & 0.256 \\
USI & & 0.69 \\
\hline
\end{tabular}

BMI: body mass index; PT: prothrombin time; INR: international normalized ratio; MEDS score: Mortality in Emergency Department Sepsis score; APACHE II score: Acute Physiology and Chronic Health Evaluation II score; IAI: intra-abdominal infection; USI: urinary system infection.

measured with coagulation-based activity assay. Plasma Ddimer levels were measured with the method of immunoturbidimetric assay. Platelet count was measured on EDTA anticoagulated samples with the method of fluorescent dye laser scattering using XE-2100 blood analyzer (Sysmex Corporation, Kobe, Japan).

2.2.4. Evaluation of Side Effects of XBJ Injection. Evaluation parameters of side effects of XBJ injection included (1) changes in vital signs; (2) complete blood cell count and urine routine examination; and (3) biochemical parameters, including hepatic function (aspartate aminotransferase, alanine aminotransferase, total bilirubin, and direct bilirubin), renal function (blood urea nitrogen and creatinine), glucose, and electrolytes.

2.2.5. Outcome of the Study. The primary outcome of this study was all-cause 28-day mortality, and the survival or death of patients within 28 days was recorded.

2.3. Statistical Analysis. The results were analyzed with SPSS 16.0 software (SPSS Inc., Chicago, IL, USA). Normally distributed data were expressed as the mean \pm standard deviation and nonnormally distributed data were expressed as the median (25-75th percentiles). The Student paired $t$-test was used to compare normally distributed data between two groups, and the rank sum test was applied for nonnormally distributed data between two groups. The $\chi^{2}$ test was used to compare frequencies. All reported $P$ values were two-sided, and $P<0.05$ was considered statistically significant.

\section{Results}

3.1. Baseline Characteristics of Patients. This study included 171 severe septic patients with DIC: 83 patients in the control group and 88 patients in the intervention group. The baseline characteristics of the overall study population are shown in Table 2. Differences of demographical data between the two groups were not statistically significant $(P$ all $>0.05)$ (Table 2$)$. In addition, the MEDS score and APACHE II score at baseline did not differ significantly between the two groups, and similar results were also found for coagulation parameters at baseline $(P$ all $>0.05)$ (Table 2$)$.

3.2. Incidence of DIC at 7 Days after Administration of XBJ Injection. Compared with the control group, incidence of DIC in the intervention group was significantly lower at 7 days after administration of XBJ injection $(P<0.001)$ (Table 3).

3.3. Clinical Severity Scores and Coagulation Parameters at 7 Days after Administration of XBJ Injection. Compared with the control group, the MEDS score was lower in the intervention group $(P=0.039)$, and the APACHE II score did not differ significantly between the two groups at 7 days after administration of XBJ injection $(P=0.107)$ (Table 3$)$. In addition, $\mathrm{PT}$ was shorter in the intervention group $(P=$ 0.003 ), and another four coagulation parameters did not differ significantly between the two groups at 7 days after administration of XBJ injection $(P$ all $>0.05)$ (Table 3$)$.

3.4. Differences of Clinical Severity Scores and Coagulation Parameters (Values at Baseline Minus Values at 7 Days after Administration of XBJ Injection). Differences of the MEDS score and APACHE II score were greater in the intervention group than in the control group $(P$ all $<0.05)$ (Table 4$)$. In addition, differences of platelet count and PT were greater in the intervention group $(P$ all $<0.05)$, and differences of another three coagulation parameters were not significant between two groups ( $P$ all $>0.05$ ) (Table 4$)$. 
TABLE 3: Incidence of DIC, clinical severity scores, and coagulation parameters at 7 days after administration of Xuebijing injection.

\begin{tabular}{lccc}
\hline & Control group $(n=83)$ & Intervention group $(n=88)$ & $P$ \\
\hline DIC $(n, \%)$ & $22(26.51)$ & $5(5.68)$ & $<0.001$ \\
Platelet $\left(\times 10^{9} / \mathrm{L}\right)$ & $119.15 \pm 91.24$ & $131.95 \pm 88.36$ & 0.205 \\
PT $(\mathrm{s})$ & $21.52 \pm 14.81$ & $17.2 \pm 8$ & 0.003 \\
Fibrinogen $(\mathrm{g} / \mathrm{L})$ & $3.65 \pm 1.63$ & $3.67 \pm 1.56$ & 0.183 \\
D-dimer $(\mathrm{mg} / \mathrm{L})$ & $3.72(1.26-6.37)$ & $3.45(1.12-5.87)$ & 0.743 \\
INR & $1.65 \pm 0.83$ & $1.65 \pm 0.93$ & 0.997 \\
MEDS score & $8(5-18)$ & $7(4-15)$ & 0.039 \\
APACHE II score & $14(11-27)$ & $13(10-25)$ & 0.107 \\
\hline
\end{tabular}

DIC: disseminated intravascular coagulation; MEDS score: Mortality in Emergency Department Sepsis score; APACHE II score: Acute Physiology and Chronic Health Evaluation II score; PT: prothrombin time; INR: international normalized ratio.

TABLE 4: Differences of clinical severity scores and coagulation parameters (values at baseline minus values at 7 days after administration of Xuebijing injection).

\begin{tabular}{lccr}
\hline & Control group $(n=83)$ & Intervention group $(n=88)$ & $P$ \\
\hline Platelet $\left(\times 10^{9} / \mathrm{L}\right)$ & $-38.95 \pm 76.06$ & $-61.44 \pm 81.84$ & 0.005 \\
PT $(\mathrm{s})$ & $4.25 \pm 6.32$ & $5.46 \pm 7.36$ & 0.036 \\
Fibrinogen $(\mathrm{g} / \mathrm{L})$ & $-0.99 \pm 4.38$ & $-1.11 \pm 4.08$ & 0.563 \\
D-dimer $(\mathrm{mg} / \mathrm{L})$ & $1.24 \pm 2.02$ & $1.63 \pm 2.21$ & 0.138 \\
INR & $0.16 \pm 0.65$ & $0.08 \pm 0.73$ & 0.472 \\
MEDS score & $2.05 \pm 3.98$ & $3.85 \pm 4.63$ & 0.007 \\
APACHE II score & $7.18 \pm 8.97$ & $10.63 \pm 9.17$ & 0.014 \\
\hline
\end{tabular}

MEDS score: Mortality in Emergency Department Sepsis score; APACHE II score: Acute Physiology and Chronic Health Evaluation II score; PT: prothrombin time; INR: international normalized ratio.

3.5. 28-Day Mortality. Compared with the control group, 28-day mortality was significantly lower in the intervention group $(P=0.034)$ (Table 2$)$.

3.6. Side Effects of XBJ Injection. There were no records of side effects during administration of XBJ injection.

\section{Discussion}

The main findings of this study were as follows: (1) compared with the control group, incidence of DIC was significantly lower in the intervention group at 7 days after administration of XBJ injection; and (2) 28-day mortality was significantly lower in the intervention group than in the control group.

$\mathrm{XBJ}$ injection is a compound preparation made from five traditional Chinese medicines. Consistency in the quality of $\mathrm{XBJ}$ injection among different batches is ensured by fingerprint technology, which refers to the use of spectroscopy and chromatography to obtain the characteristics of component groups, maps, or images, combined with computer technology to analyze information, thereby identifying the authenticity of the drugs [19]. Multiple bioactive constituents, such as safflower yellow A, ferulic acid, and Danshensu, are identified in XBJ injection, and these constituents are responsible for the therapeutic effects of XBJ injection [20, 21].

Currently, there are few studies on XBJ injection for treating DIC. Furthermore, the dose and time of XBJ injection in previous studies were not uniform. The report of Guo et al. included 4 patients with DIC, among whom two received $100 \mathrm{~mL} \mathrm{XBJ}$ injection twice daily, one received $100 \mathrm{~mL} \mathrm{XBJ}$ injection once daily, and one received $200 \mathrm{~mL} \mathrm{XBJ}$ injection twice daily [13]. In Chen's study, 30 patients with DIC received $100 \mathrm{~mL}$ XBJ injection twice daily, but the administration time ranged from 7 to 14 days [14]. According to the instructions of this drug, $100 \mathrm{~mL} \mathrm{XBJ}$ injection with $100 \mathrm{~mL}$ normal saline twice daily for 7 consecutive days is recommended for patients with organ dysfunction caused by infection; on the other hand, three or four times daily administration is allowed, and administration time may be ranged from 7 to 14 days for very critically ill patients. On the basis of these recommendations, the routine dose and time of $\mathrm{XBJ}$ injection for patients with severe sepsis are $100 \mathrm{~mL} \mathrm{XBJ} \mathrm{injection} \mathrm{with}$ $100 \mathrm{~mL}$ normal saline twice daily for 7 consecutive days in our EICU. Therefore, this dose and time were selected in this study.

It has been recognized that the crosstalk between inflammation and coagulation is important in the pathogenesis of sepsis, and endothelium is the central link. During sepsis, inflammatory mediators cause endothelial damage, and damaged endothelium manifests enhanced procoagulatory properties and leads to coagulation activation; vice versa coagulation activation enhances inflammatory responses. DIC is the most severe form of coagulation abnormalities and its development is closely associated with endothelial damage [2]. Previous clinical studies have confirmed that XBJ injection can alleviate the extent of endothelial damage in 
critically ill patients $[22,23]$. In addition, XBJ injection can inhibit coagulation activation $[24,25]$ and reduce inflammatory responses $[26,27]$. In this study, we found that, compared with the control group, incidence of DIC was significantly lower in the intervention group at 7 days after administration of XBJ injection (Table 3). Furthermore, differences of platelet count and PT between values at baseline and 7 days after administration of XBJ injection were significantly greater in the intervention group (Table 4). Taken together, the therapeutic effects of XBJ injection on DIC may be attributed to its protective roles on the endothelium and are mainly dependent on increasing platelet count and decreasing PT.

The MEDS score and APACHE II score at baseline were not significantly different between the two groups (Table 2), indicating that the disease severity of patients in the two groups was comparable before treatment. However, after treatment, the MEDS score was significantly lower in the intervention group than in the control group (Table 3). Furthermore, differences of the MEDS score and APACHE II score between values at baseline and 7 days after administration of XBJ injection were significantly greater in the intervention group (Table 4). These results demonstrate that $\mathrm{XBJ}$ injection may obviously alleviate the disease severity in severe septic patients with DIC. Previous studies have shown that incidence of DIC increases with the severity of sepsis $[28,29]$. Therefore, these results may be associated with the effects of XBJ injection on DIC.

28-day mortality in the intervention group and control group was $20.45 \%$ and $34.94 \%$, respectively. It is notable that 28-day mortality was significantly lower in the intervention group (Table 2). Previous studies have demonstrated that mortality in septic patients with DIC is significantly higher than in those without DIC, and resolution of DIC is associated with a favorable outcome $[30,31]$. In the present study, incidence of DIC was significantly lower after administration of XBJ injection, confirming that this drug is effective in improving short-term prognosis of severe septic patients with DIC.

An important limitation of this study is the retrospective design. Although the data were analyzed on the basis of laboratory parameters at entry and after treatments, this type of analysis should always be interpreted with care. Another important limitation is that this was a single-center study, and the results may not be applicable to other hospitals. Therefore, a prospective, multicenter, randomized controlled trial is needed to further confirm the effects of XBJ injection in severe septic patients with DIC in future research.

\section{Conclusions}

In conclusion, this study demonstrates that XBJ injection can effectively treat DIC caused by severe sepsis and improve short-term prognosis of severe septic patients with DIC.

\section{Conflict of Interests}

The authors declare that there is no conflict of interests regarding the publication of this paper.

\section{References}

[1] D. C. Angus, W. T. Linde-Zwirble, J. Lidicker, G. Clermont, J. Carcillo, and M. R. Pinsky, "Epidemiology of severe sepsis in the United States: analysis of incidence, outcome, and associated costs of care," Critical Care Medicine, vol. 29, no. 7, pp. 1303-1310, 2001.

[2] M. Levi and H. ten Cate, "Disseminated intravascular coagulation," The New England Journal of Medicine, vol. 341, no. 8, pp. 586-592, 1999.

[3] S. Zeerleder, C. E. Hack, and W. A. Wuillemin, "Disseminated intravascular coagulation in sepsis," Chest, vol. 128, no. 4, pp. 2864-2875, 2005.

[4] R. L. Bick, "Disseminated intravascular coagulation current concepts of etiology, pathophysiology, diagnosis, and treatment," Hematology/Oncology Clinics of North America, vol. 17, no. 1, pp. 149-176, 2003.

[5] N. Sakuragawa, H. Hasegawa, M. Maki, M. Nakagawa, and M. Nakashima, "Clinical evaluation of low-molecular-weight heparin (FR-860) on disseminated intravascular coagulation (DIC) - a multicenter co-operative double-blind trial in comparison with heparin," Thrombosis Research, vol. 72, no. 6, pp. 475-500, 1993.

[6] N. Aoki, T. Matsuda, H. Saito et al., "A comparative doubleblind randomized trial of activated protein $\mathrm{C}$ and unfractionated heparin in the treatment of disseminated intravascular coagulation," International Journal of Hematology, vol. 75, no. 5, pp. 540-547, 2002.

[7] G. R. Bernard, J.-L. Vincent, P.-F. Laterre et al., "Efficacy and safety of recombinant human activated protein $\mathrm{C}$ for severe sepsis," The New England Journal of Medicine, vol. 344, no. 10, pp. 699-709, 2001.

[8] J.-F. Dhainaut, S. B. Yan, D. E. Joyce et al., "Treatment effects of drotrecogin alfa (activated) in patients with severe sepsis with or without overt disseminated intravascular coagulation," Journal of Thrombosis and Haemostasis, vol. 2, no. 11, pp. 1924-1933, 2004.

[9] V. M. Ranieri, B. T. Thompson, P. S. Barie et al., "Drotrecogin alfa (activated) in adults with septic shock," The New England Journal of Medicine, vol. 366, no. 22, pp. 2055-2064, 2012.

[10] R. P. Dellinger, M. M. Levy, A. Rhodes et al., "Surviving sepsis campaign: international guidelines for management of severe sepsis and septic shock: 2012," Critical Care Medicine, vol. 41, no. 2, pp. 580-637, 2013.

[11] P. Jia, S. Wang, X. Meng et al., "Effects of ionic liquid and nanogold particles on high-performance liquid chromatography-electrochemical detection and their application in highly efficient separation and sensitive analysis of five phenolic acids in Xuebijing injection," Talanta, vol. 107, pp. 103-110, 2013.

[12] N. Li, L. W. Jiang, L. Yu et al., "Systematic review of Xuebijing injection for the treatment of sepsis," Chinese Journal of Modern Drug Application, vol. 7, no. 22, pp. 8-11, 2013.

[13] N. Guo, Q. Q. Liu, Q. Miao, and Q.-M. Jiang, "Effects of Xuebijing injection in 4 patients with disseminated intravascular coagulation," Chinese Critical Care Medicine, vol. 19, no. 7, p. 441, 2007.

[14] Z. Y. Chen, "Effects of Xuebijing injection in 30 patients with disseminated intravascular coagulation," Shanxi Medical Journal, vol. 37, no. 6, pp. 732-733, 2008. 
[15] M. M. Levy, M. P. Fink, J. C. Marshall et al., "2001 SCCM/ ESICM/ACCP/ATS/SIS International Sepsis Definitions Conference," Critical Care Medicine, vol. 31, no. 4, pp. 1250-1256, 2003.

[16] F. B. Taylor Jr., C.-H. Toh, W. K. Hoots, H. Wada, and M. Levi, "Towards definition, clinical and laboratory criteria, and a scoring system for disseminated intravascular coagulation: on behalf of the scientific subcommittee on Disseminated Intravascular Coagulation (DIC) of the International Society on Thrombosis and Haematostasis (ISTH)," Thrombosis and Haemostasis, vol. 86, no. 5, pp. 1327-1330, 2001.

[17] N. I. Shapiro, R. E. Wolfe, R. B. Moore, E. Smith, E. Burdick, and D. W. Bates, "Mortality in Emergency Department Sepsis (MEDS) score: a prospectively derived and validated clinical prediction rule," Critical Care Medicine, vol. 31, no. 3, pp. 670675, 2003.

[18] W. A. Knaus, E. A. Draper, D. P. Wagner, and J. E. Zimmerman, "APACHE II: a severity of disease classification system," Critical Care Medicine, vol. 13, no. 10, pp. 818-829, 1985.

[19] G. A. Luo and Y. M. Wang, "Classification and development of traditional Chinese medicine fingerprint," Chinese Journal of New Drugs, vol. 11, no. 1, pp. 46-51, 2002.

[20] H. Huang, L. Ji, S. Song et al., "Identification of the major constituents in Xuebijing injection by HPLC-ESI-MS," Phytochemical Analysis, vol. 22, no. 4, pp. 330-338, 2011.

[21] M. Jiang, M. Zhou, Y. Han et al., "Identification of NF- $\kappa$ B Inhibitors in Xuebijing injection for sepsis treatment based on bioactivity-integrated UPLC-Q/TOF," Journal of Ethnopharmacology, vol. 147, no. 2, pp. 426-433, 2013.

[22] J. J. Dai, S. H. Cao, and J. D. Wang, "The protective effect of Xuebijing on the vascular endothelium of critical patients," Chinese General Practice, vol. 8, no. 18, pp. 1486-1487, 2005.

[23] M. Chen, A. H. Fei, W. W. Lu et al., "Clinical research on the therapeutic effect of Xuebijing injection on sepsis and its influence on PECAM-1 and coagulation function," Modern Journal of Integrated Traditional Chinese and Western Medicine, vol. 21, no. 11, pp. 1156-1158, 2012.

[24] L. Q. Tang, N. Peng, Z. G. Pan, H. Tong, and L. Su, "The effect of Xuebijing injection on the coagulation parameters and outcome of the patients with severe sepsis," Chinese Journal of Integrated Traditional Chinese and Western Medicine in Critical Care, vol. 19, no. 3, pp. 132-136, 2012.

[25] L. D. Zhang and X. J. Pei, "Effects of Xuebijing parenteral solution on blood coagulation function and blood gas analysis in severe sepsis patients," Chinese Journal of Critical Care Medicine, vol. 32, no. 12, pp. 1088-1091, 2012.

[26] F. Qi, Z.-X. Liang, D.-Y. She, G.-T. Yan, and L.-A. Chen, "A clinical study on the effects and mechanism of Xuebijing injection in severe pneumonia patients," Journal of Traditional Chinese Medicine, vol. 31, no. 1, pp. 46-49, 2011.

[27] Y. L. Song, Z. J. Song, J. J. Jiang et al., "Clinical efficacy and safety evaluation of Xuebijing on severe pneumonia," International Journal of Respiration, vol. 32, no. 22, pp. 1692-1695, 2012.

[28] S. Gando, D. Saitoh, H. Ogura et al., "Natural history of disseminated intravascular coagulation diagnosed based on the newly established diagnostic criteria for critically ill patients: results of a multicenter, prospective survey," Critical Care Medicine, vol. 36, no. 1, pp. 145-150, 2008.

[29] K. Okabayashi, H. Wada, S. Ohta, H. Shiku, T. Nobori, and K. Maruyama, "Hemostatic markers and the sepsis-related organ failure assessment score in patients with disseminated intravascular coagulation in an intensive care unit," American Journal of Hematology, vol. 76, no. 3, pp. 225-229, 2004.

[30] P. Cauchle, C. Cauchie, K. Z. Boudjeltia et al., "Diagnostic and prognosis of overt disseminated intravascular coagulation in a general hospital-meaning of the isth score system, fibrin monomers, and lipoprotein-C-reactive protein complex formation," American Journal of Hematology, vol. 81, no. 6, pp. 414419, 2006.

[31] J. Kienast, M. Juers, C. J. Wiedermann et al., “Treatment effects of high-dose antithrombin without concomitant heparin in patients with severe sepsis with or without disseminated intravascular coagulation," Journal of Thrombosis and Haemostasis, vol. 4, no. 1, pp. 90-97, 2006. 


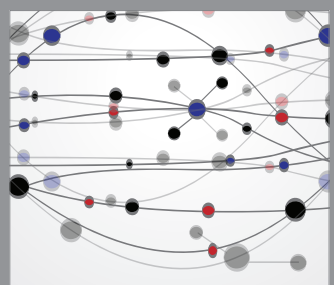

The Scientific World Journal
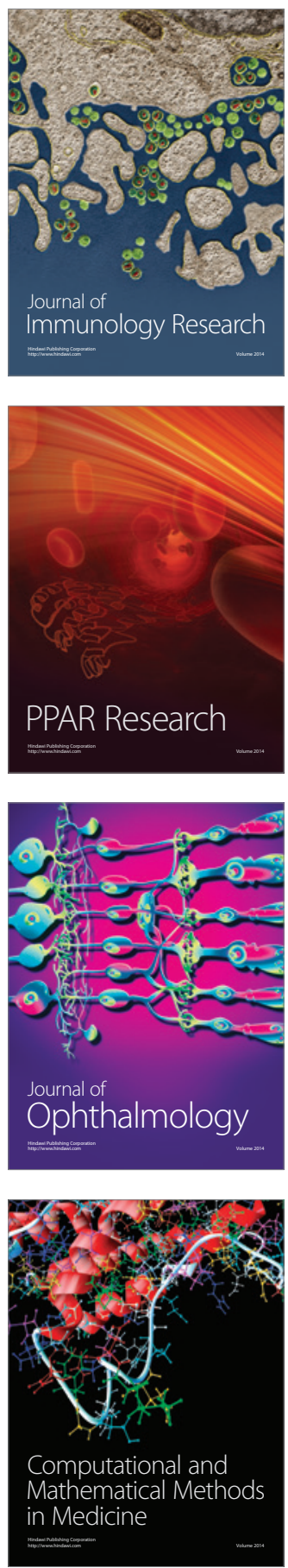

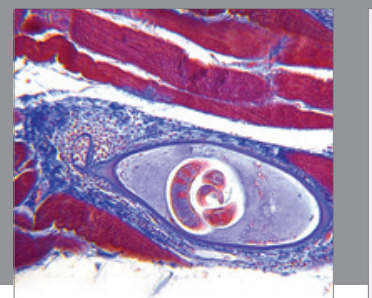

Gastroenterology

Research and Practice
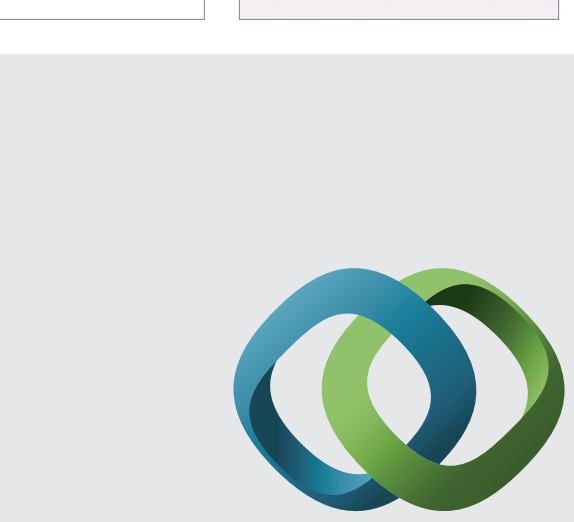

\section{Hindawi}

Submit your manuscripts at

http://www.hindawi.com
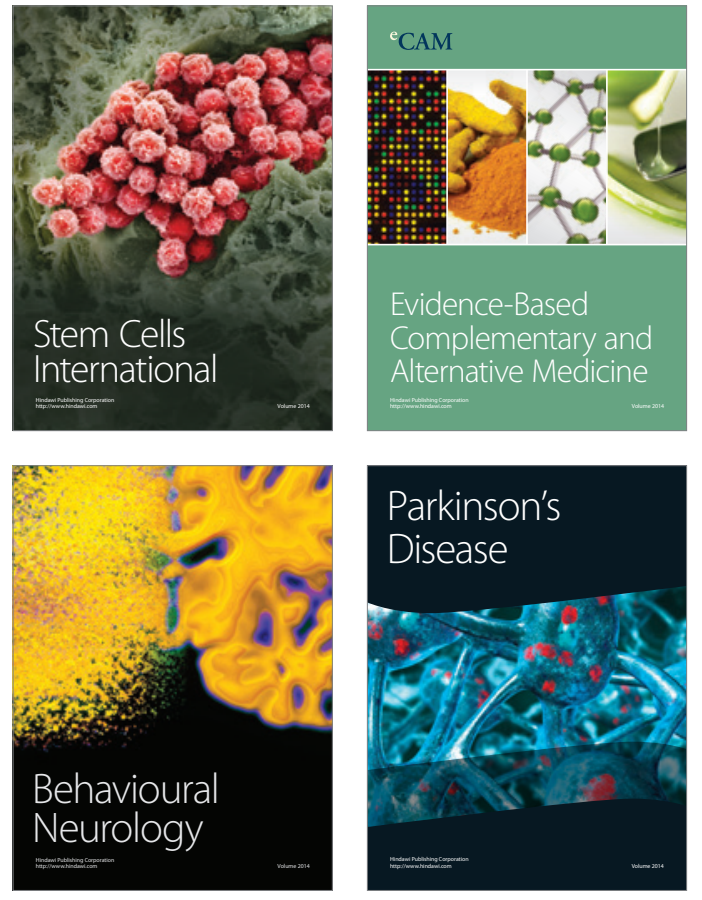
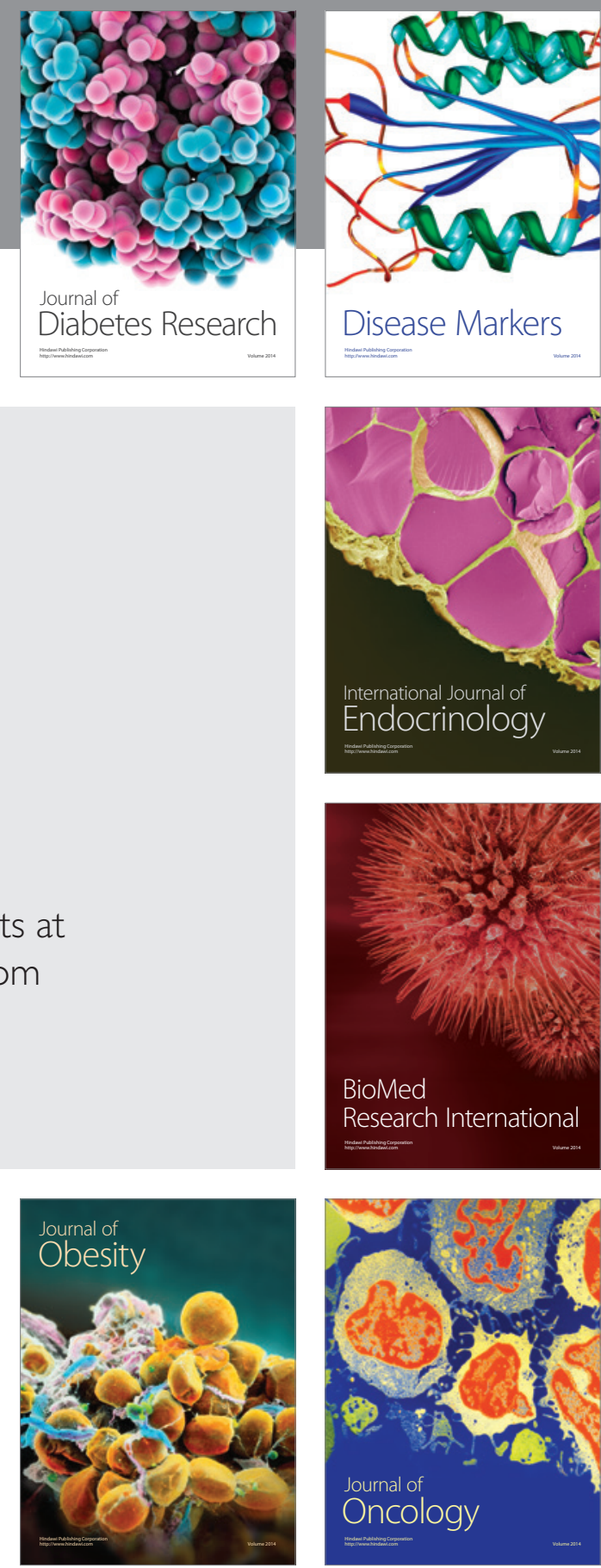

Disease Markers
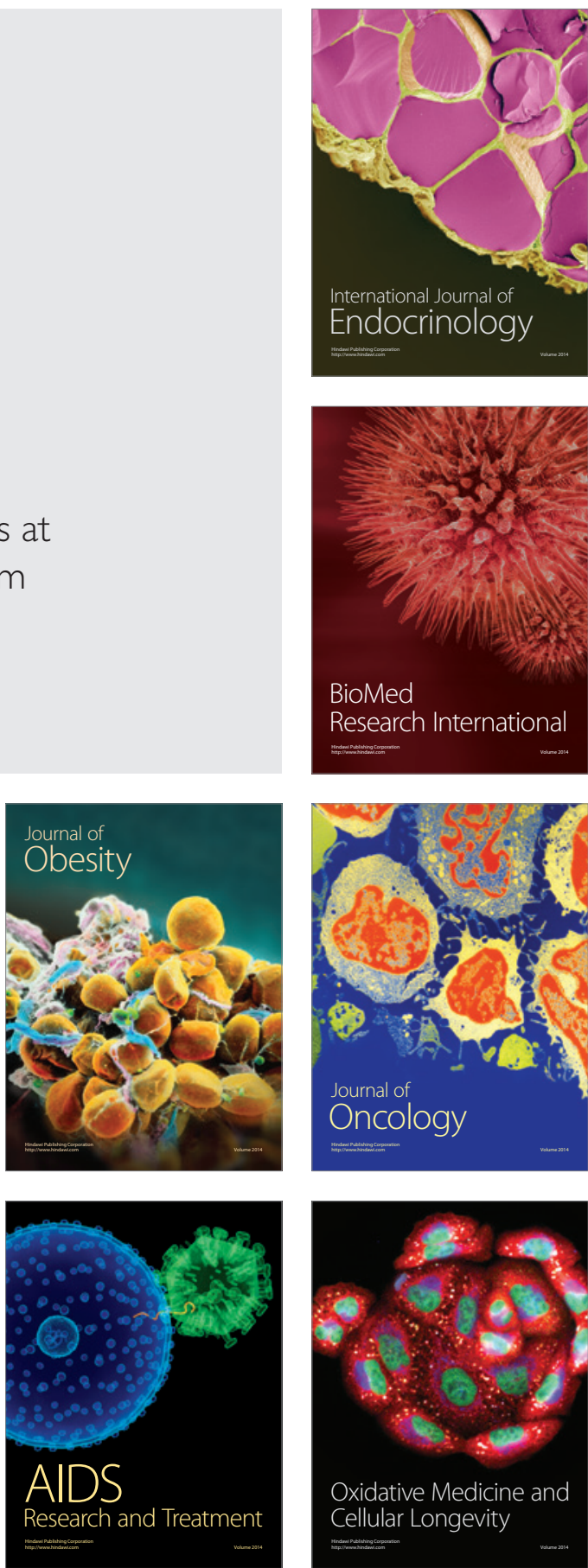